\title{
Comparison and evaluation of three different molecular methods for detection of human Betapapillomaviruses in skin biopsies from patients with nonmelanoma skin cancer and precancerous lesions
}

\author{
Jolanta Kopec ${ }^{1}$, Slawa Szostek1, Joanna Sulowicz² and Barbara Zawilinska1 \\ 1Jagiellonian University Medical College, Faculty of Medicine, Chair of Microbiology, Department of Virology, Kraków, Poland; ${ }^{2}$ Jagiellonian Uni- \\ versity Medical College, Faculty of Medicine, Department of Dermatology, Kraków, Poland
}

\begin{abstract}
Betapapillomaviruses have been linked to the development of nonmelanoma skin cancers. A great diversity of these viruses in skin specimens requires the use of sensitive and reliable detection methods. There are currently no standardized assays for diagnostic purposes. A combination of several molecular methods has great practical significance and gives the opportunity to broaden the spectrum of detected Beta-HPV types. In the present study, different molecular methods for Beta-HPVs detection and genotyping were used: PCRs with different sets of primers, PCR followed by reverse hybridization and direct sequencing of PCR amplimers; all performed in skin biopsies from lesions and perilesional healthy area of 118 patients with NMSC or precancerous lesions. Beta-HPVs were detected in $41 \%$ of 261 biopsies examined. The RHA for 25 types of Beta-HPVs showed a significantly higher sensitivity than PCR-based methods and allowed to detect 172 genotypes in 86 samples, including 39 with multiple infections. The most frequently identified types were HPV23, HPV24 and HPV93. HPV5 and HPV8, considered high-risk carcinogen types, were detected only in a small percentage of samples. Direct sequencing confirmed the presence of Beta-HPV genotypes from outside of RHA panel in the analysed biopsies. This allowed detecting thirty-two additional genotypes in 5 samples, that were positive only in RHA with the universal probe, which failed to identify the virus genotypes. Our findings confirmed the need to apply different methods to detect Beta-HPV infections.
\end{abstract}

Key words: Betapapillomaviruses, skin tumour, Beta-HPV detection, genotyping, reverse hybridization, sequencing

Received: 13 February, 2020; revised: 20 February, 2020; accepted: 08 April, 2020; available on-line: 08 June, 2020

⿶e-mail: jolanta.kopec@uj.edu.pl

Funding: This study was partially funded by the Polish Committee for Scientific Research (Grant N402 1861 35).

Abbreviations: HPV, Human Papillomavirus; Beta-HPVs, types of the genus Betapapillomavirus, beta-1 to beta-5, five species of the genus Betapapillomavirus; EV, epidermodysplasia verruciformis; SCC, squamous cell carcinoma; BCC, basal cell carcinoma; NMSC, nonmelanoma skin cancer; nPCR, nested PCR; RHA, reverse hybridization assay

\section{INTRODUCTION}

HPVs that infect cutaneous or mucosal epithelia belong to the Papillomaviridae family of DNA viruses. Based on their DNA sequence, a phylogenetic tree was generated, in which the different HPV types are classified into five genera, named with Greek letters: Alpha-, Beta-, Gamma-, $M u$ - and Nupapillomavirus (Tommasino, 2017; Van Doorslaer et al., 2018). The genus Alphapapillomavirus contains many HPV types related to the neoplastic processes in the genital tract (Zur Hausen, 2000; Szostek et al., 2008). Beta-HPVs have an affinity for the skin. More than 50 fully sequenced and characterized types of human Beta-HPVs are further subdivided into five different species (beta-1 to beta-5) (Van Doorslaer et al., 2018; Gheit, 2019). The HPV5 and HPV8 types from the beta-1 species, previously known as EV-associated types, were first identified in warts and skin carcinomas of EV patients (Jablonska et al., 1972; de Jong et al., 2018). These two types have been very often found in SCC samples obtained from patients with $\mathrm{EV}$ and thus could be qualified as high-risk types (carcinogen group 2B) (Bouvard et al., 2009; Viarisio et al., 2017). The important role of Beta-HPVs in NMSC in non-EV individuals is still being discussed. Cutaneous HPVs are characterized by a great diversity; they are ubiquitous and widespread in the general population. The infections can be persistent and asymptomatic, often caused by many types of BetaHPVs, but the viral load for an individual type can be very low (Forslund, 2007; Rollison et al., 2019; Tommasino, 2019). Moreover, there are no standardized diagnostics assays and one commercially available test for genotyping is only used for research purposes. In the face of the above facts, early and reliable diagnostics is particularly important.

The study of the association between Beta-HPVs and NMSC depends on HPV detection and genotyping methods (Meyer et al., 2000; Stockfleth et al., 2004). Various studies used different diagnostic methods. The leading assays are those based on molecular techniques, such as PCR with different modifications, followed by genotyping by sequencing of the PCR products, reverse line blotting assay, restriction fragment length polymorphism analysis or hybridization with oligonucleotide probes (Antonsson et al., 2013; Bouwes Bavinck et al., 2010; Brink et al., 2005).

The family of skin-tropic HPVs is very diversified and for this reason, several primer sets were described and analyzed in previous years, e.g. the consensus degenerate CP65/70 and CP66/69, FAP59/64 and FAP6085/6319 or CP62/71 and CP64/70 for the nPCR (Berkhout et al., 1995; Berkhout et al., 2000; Forslund et al., 2003) as well as the consensus nondegenerate or specific primers (PM primers or primers for multiplex PCR) recommended by de Koning and others (de Koning et al., 2006) and Gheit 


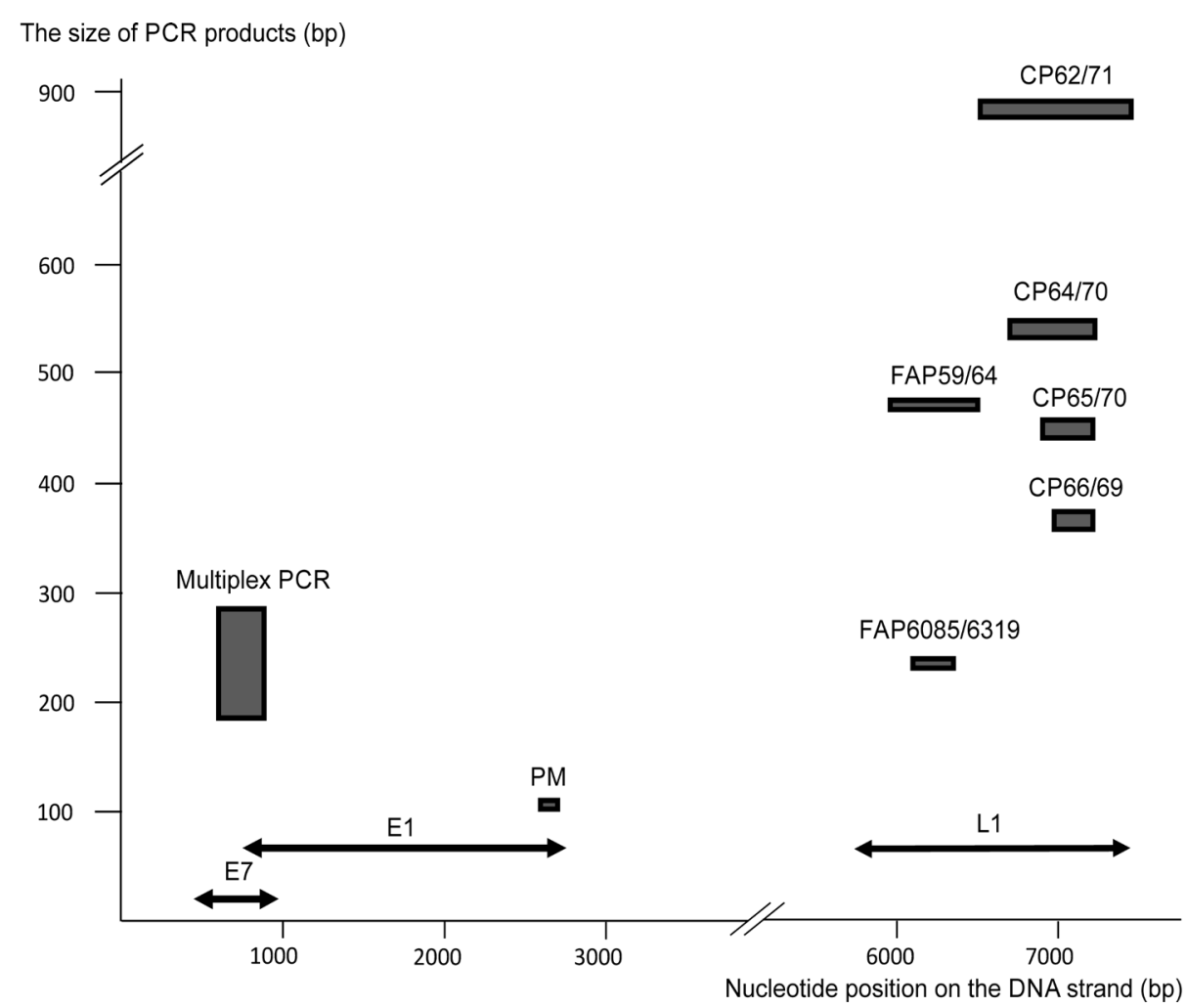

Figure 1. Simplified chart of the size and position of the PCR products in reference to E1, E7 and L1 HPV8 genes location on the DNA strand

The size and position of the PCR products for different primers according to Berkhout et al., 1995, 2000; Forslund et al., 2003; de Koning et al., 2006; Gheit et al., 2007.

and others (Gheit et al., 2007), respectively. With their specific amplification conditions, these primers targeted different genes of various types of cutaneous HPVs and allowed the detection of a wide range of Beta-HPVs. Figure 1 shows various attachment sites of the aforementioned primer sets and the size of the amplification products obtained by various methods. The main disadvantage of the nPCRs introduced above is their limited sensitivity to distinct HPV types as well as a possibility of contamination associated with nPCR procedures and a higher risk of nonspecific cross-reactions (de Koning et al., 2006). The introduction of single-type specific primers (Meyer et al., 2000; Pfister et al., 2003) results in higher sensitivity, but encompasses only selected HPV types and therefore is very laborious.

The aim of the present study was to determine the prevalence of Beta-HPV DNA in patients with NMSC or precancerous lesions. Various molecular methods for detection of Beta-HPV sequences such as: PCR with general and specific primers as well as direct sequencing of nPCR products and PCR combined with reverse hybridization of biotinylated amplicons for genotyping of Beta-HPVs were used. These methods were evaluated for their suitability for the identification of cutaneous HPVs in biopsies from changed and unchanged skin. It was assumed that the combination of several diagnostic tests may give the opportunity to broaden the spectrum of detected types. This may be crucial in view of the diagnostic difficulties in detecting Beta-HPVs.

\section{MATERIALS AND METHODS}

A total of 261 biopsies were collected from 106 patients with NMSC and 12 patients with precancerous skin lesions. Among the individuals with NMSC, there were 95 patients with histopathologically confirmed BCC and 11 with SCC. Patients were treated between 2006 and 2016 in the Department of Dermatology, Jagiellonian University Medical College, Krakow, Poland. The mean age of patients was 71.7 years (range of 33-96 years). Men predominated $(73 / 118 ; 61.9 \%)$ in the studied group.

Biopsies were taken not only from tumours but also from perilesional healthy skin of each patient to assess the presence of HPVs. In 12 patients, specimens were taken from two to three different locations. All samples were collected using sterile scalpels and then immersed in $0.9 \% \mathrm{NaCl}$. They were frozen at $-80^{\circ} \mathrm{C}$ in individual Eppendorf tubes and successively tested. The study was approved by the Bioethics Committee of the Jagiellonian University (KBET/100/B/2006; KBET/214/B/2013; $\mathrm{KBET} / 108 / \mathrm{B} / 2014)$, and informed consent was obtained from each patient.

DNA isolation. DNA was isolated from each specimen using the spin-column method (Genomic Mini Test, A\&A Biotechnology, Poland) according to the manufacturer's instruction. To determine the presence and the quality of human DNA in the samples, the beta-actin gene amplification was performed resulting in a $307 \mathrm{bp}$ product, according to the procedure described earlier (Szostek et al., 2009).

Detection of cutaneous Beta-HPVs by PCR. The nPCR method, according to Berkhout et al. (1995), was used as the first step for the preliminary examination. In this reaction, two pairs of general degenerate primers CP65-70 (CP65/70 and CP66/69 as external and internal, respectively) were used for the detection of EV-associated HPVs (HPV5, 8, 9, 12, 14, 15, 17, 19, 20, 
Table 1. Comparison of results obtained by different methods used for the detection of human Beta-HPVs in 261 skin biopsies

\begin{tabular}{|c|c|c|c|c|c|c|}
\hline Methods & Both positive & $\begin{array}{l}\text { Both } \\
\text { negative }\end{array}$ & Pos./neg. & Neg./pos. & $\begin{array}{l}\text { Overall } \\
\text { agreement }\end{array}$ & $\begin{array}{l}\text { Pearson's } \\
\text { correlation coefficient }\end{array}$ \\
\hline $\mathrm{nPCR}$ vs. RHA universal probe & 51 & 160 & 7 & 43 & $80.8 \%$ & $0.58(p=0.00)$ \\
\hline nPCR vs. RHA totala & 52 & 156 & 6 & 47 & $79.9 \%$ & $\begin{array}{l}0.57 \\
(p=0.00)\end{array}$ \\
\hline $\begin{array}{l}\text { RHA universal probe vs. RHA } \\
\text { specific probes }\end{array}$ & 81 & 162 & 13 & 5 & $93.1 \%$ & $\begin{array}{l}0.85 \\
(p=0.00)\end{array}$ \\
\hline $\begin{array}{l}\text { HPV5 RHA genotyping vs. PCR } \\
\text { spec. HPV5 }\end{array}$ & 8 & 248 & 2 & 3 & $98.1 \%$ & $\begin{array}{l}0.75 \\
(p=0.00)\end{array}$ \\
\hline $\begin{array}{l}\text { HPV8 RHA genotyping vs. PCR } \\
\text { spec. HPV8 }\end{array}$ & 3 & 253 & 4 & 1 & $98.1 \%$ & $\begin{array}{l}0.56 \\
(p=0.00)\end{array}$ \\
\hline nPCR vs. RHA EV-specific probes ${ }^{b}$ & 31 & 169 & 27 & 34 & $76.6 \%$ & $\begin{array}{l}0.35 \\
(p=0.000)\end{array}$ \\
\hline $\begin{array}{l}\text { nPCR vs. RHA nonEV-specific } \\
\text { probesc }\end{array}$ & 27 & 188 & 31 & 15 & $82.4 \%$ & $\begin{array}{l}0.44 \\
(p=0.000)\end{array}$ \\
\hline
\end{tabular}

aresults of RHA with universal and specific probes; 'bHA EV, specific probes for genotypes: HPV5, 8, 9, 12, 14, 15, 17, 19, 20, 21, 22, 23, 24, 25, 36, 37, 38, 49; 'RHA nonEV, specific probes for genotypes: HPV47, 75, 76, 80, 92, 93, 96; EV, epidermodysplasia verruciformis

$21,22,23,24,25,36,37,38,46,49)$. Separate PCR reactions with primers specific for HPV5 (Kawase et al., 1996) and HPV8 (Fuchs et al., 1986) were also applied. Fifty ng of genomic DNA was used as the template. All amplifications were performed using the S1000 thermal cycler (Biorad, USA). For every reaction, negative (water) and positive controls were included. The positive controls were obtained by courtesy of Prof. M. Malejczyk from the Medical University of Warsaw, Poland. The amplified products were analyzed by electrophoresis in $3 \%$ agarose gel and stained with ethidium bromide.

The analytical sensitivity of the nPCR was determined by amplification of ten-fold serial dilutions of the positive control (HPV5).

PCR with RHA. Detection and genotyping of 25 different types (HPV5, 8, 9, 12, 14, 15, 17, 19, 20, 21, 22, $23,24,25,36,37,38,47,49,75,76,80,92,93,96)$ of cutaneous Beta-HPVs from five species was carried out with RHA Kit Skin (beta) HPV (Diassay, Rijswijk, The Netherlands). This panel included both the EV-associated and non-EV-associated HPV types. In this method, a one-step amplification of a 117 -base pair region from the E1 gene of Beta-HPVs was applied using the nondegenerate PM primer set containing a biotin label. Bioti- nylated amplicons were hybridized with oligonucleotide probes immobilized as parallel lines on membrane strips. The hybrids formed were visualized in an enzymatic colour reaction. Positive (HPV93 plasmid clone) and negative (water) controls were included in all reactions.

In addition to specific probes, each strip contained a mixture of universal HPV probes for the detection of almost all Beta-HPVs. Positive results with those universal probes without manifestation of specific types indicated the presence of other genotypes from the Beta-HPVs group. All procedures were performed exactly according to the manufacturer's instructions.

Sequencing. To determine the Beta-HPV types in selected samples, the amplification products derived from EV-spectrum nPCR were purified and analyzed by direct DNA sequencing performed on the automated $3730 \mathrm{xl}$ DNA Analyzer system (Applied Biosystems). The obtained DNA sequences were compared to all available HPV sequences stored in the NCBI database by the nucleotide-nucleotide and nucleotide-protein BLAST search program.

Statistical analysis. The obtained results were analyzed using non-parametric Pearson's Chi-squared and Fisher's exact tests as well as the Mann-Whitney U test

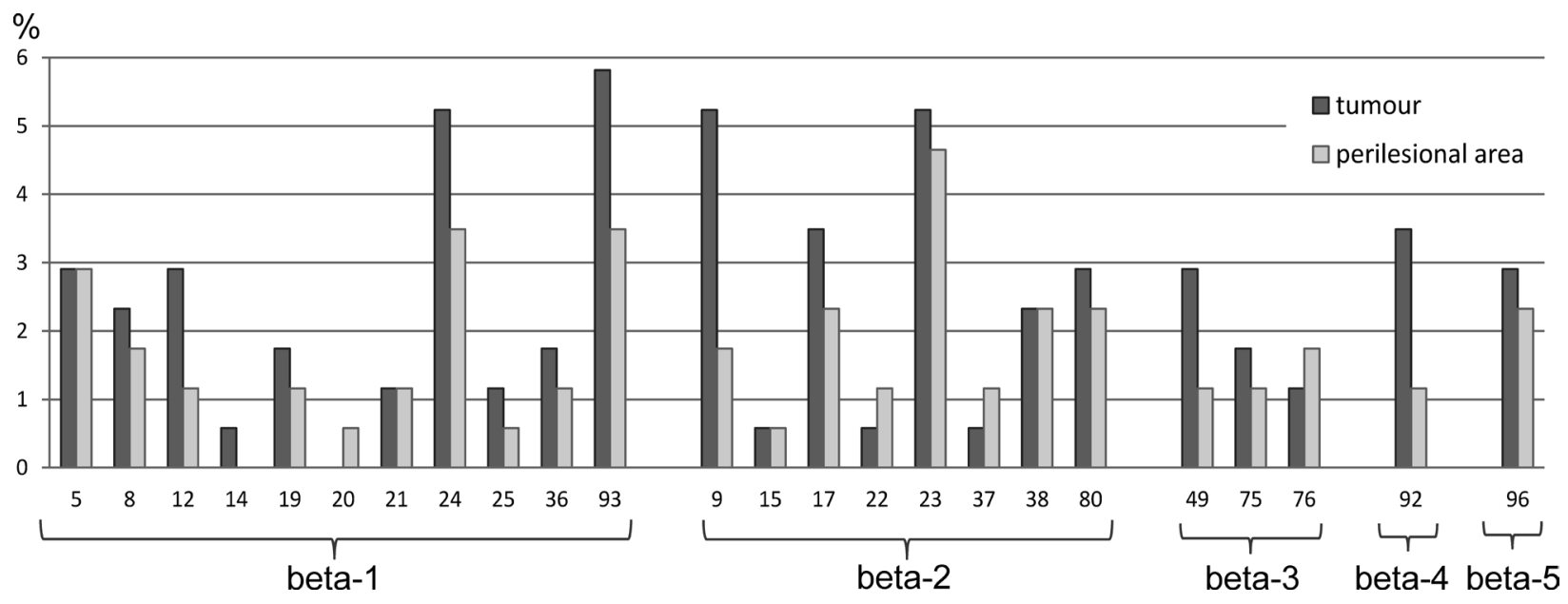

Figure 2. The spectrum and percentage of Beta-HPV genotypes in 86 samples assayed by RHA. Genotypes were classified into 5 different species according to Van Doorslaer et al., 2018 
Table 2. Results of direct sequencing of nPCR amplicons in 19 samples possible to analyse

\begin{tabular}{llll}
\hline Sequencing results & $\begin{array}{l}\text { Established types } \\
(>90 \% \text { homology })\end{array}$ & $\begin{array}{l}\text { Related types } \\
(75-90 \% \text { homology })\end{array}$ & No. of detected genotypes \\
\hline Types from the RHA panel & $15(60 \%)$ & $89(55 \%)$ & $104(56 \%)$ \\
\hline Types from outside of the RHA panel & $10(40 \%)$ & $72(45 \%)$ & $82(44 \%)$ \\
\hline Total & 25 & 161 & 186 \\
\hline
\end{tabular}

to evaluate mixed-type Beta-HPV infections. In all of the above tests, the results were statistically significant at $p<0.05$. Pearson's correlation coefficient value was categorized as follows: poor agreement $(<0.20)$, fair agreement (0.21-0.40), moderate agreement (0.41-0.60), good agreement $(0.61-0.80)$, and very good agreement $(>0.8)$. Statistical analysis was performed using the Statistica 13.3 software.

\section{RESULTS}

All examined samples were positive for beta-actin, which confirmed their suitability for further analysis. The analytical sensitivity of nPCR with our modifications was determined as 100-1000 viral copies per reaction.

Overall, all the methods described above allowed for the detection of Beta-HPVs in 106 (41\%) specimens from $64(54 \%)$ patients. Positive results were obtained by nPCR and by RHA with the universal probe in $22.2 \%(58 / 261)$ and $36.0 \%(94 / 261)$ of samples, respectively. These differences were statistically significant (Chi-squared test with Pearson's modification, $\mathrm{p}<0.001$ ). The agreement of these two methods was $80.8 \%$. The sensitivity and specificity of nPCR compared to RHA with the universal probe were $54.2 \%$ and $95.8 \%$, respectively.

The use of PCR with specific primers for HPV5 and HPV8 enabled the detection of these viruses in 11 and 4 samples, respectively, with two samples having both viruses at the same time. In 5 cases, the positive results did not coincide with nPCR.

In reverse hybridization, 99/261 (38\%) samples were positive, of which in 86 samples the Beta-HPV genotypes were identified, while in the further 13 , only hybridization with universal probes was observed. In 6 specimens with a particular genotype (HPV38, 92 and 93), simultaneous negative results with the universal probes were obtained. The results of the comparison of the above-mentioned methods were presented in Table 1.

The most frequently identified species was beta-1 $(43 \%$ of all genotypes) followed by beta-2 (37\%). The spectrum of genotypes in tumours and the perilesional area was presented in Fig. 2. In the 86 HPV-genotyped samples, 172 genotypes were identified. The most frequently identified type was HPV23 (17 samples), followed by HPV93 and HPV24 (16 and 15 samples, respectively). The HPV47 type was not found. A single genotype occurred in 47 $(54.6 \%)$ samples, while multiple infections with two or more skin HPVs (up to 8) were present in 39 samples (average $3.2 \pm 1.7$ genotypes). The most common types found in co-infections were HPV23, 24 and 9.

Among the 261 specimens, 135 samples were collected from the centre of tumours and 126 from perilesional skin without clinical changes. The analysis of these specimens demonstrated that there were no statistically significant differences between those two kinds of material in positive results using nPCR and RHA with universal probes. The average number of genotypes in the samples taken from the tumour and from healthy skin was $2.1 \pm 1.7$ and $1.9 \pm 1.4$, respectively, and no significant differences in the occurrence of single and mixed infections were observed (Mann-Whitney U test, $p=0.8$ ). Some differences were revealed only in the frequency of several HPV types, i.e.: HPV9, HPV12, HPV49, HPV92 and HPV93, which appeared slightly more often in the biopsies from lesions than in those from healthy skin. Using all mentioned tests, half of 128 sample pairs (lesion/perilesional healthy area) had negative results, while in $31.2 \%$ of the examined pairs, the presence of BetaHPV DNA was confirmed in both samples. Divergent results were obtained for 24 pairs (in 17 of them HPV was detected only in the biopsies taken from the lesions and in 7 only in the biopsies from healthy skin).

Table 3. Presentation of Beta-HPV types obtained by sequencing in five samples reacting only with the universal probe in RHA

\begin{tabular}{|c|c|c|c|c|}
\hline Sample ID & $\begin{array}{l}\text { Established } \\
\text { Beta-HPV types }\end{array}$ & $\begin{array}{l}\text { Related } \\
\text { Beta-HPV types }\end{array}$ & No. of detected types & $\begin{array}{l}\text { No. of types from } \\
\text { outside of the RHA-panel }\end{array}$ \\
\hline 10 & 124,152 & 36 & 3 & 2 \\
\hline 47 & - & $\begin{array}{l}17,20,37, \mathbf{1 1 0}, \mathbf{1 1 3} \\
\mathbf{1 2 2}, \mathbf{1 4 5}, \mathbf{1 7 4}\end{array}$ & 8 & 5 \\
\hline 162 & 20 & 21 & 2 & - \\
\hline 230 & 15 & $\begin{array}{l}9,17,38,80,110,111, \\
113,122,145,174\end{array}$ & 11 & 6 \\
\hline 240 & 5,36 & $24,93,98,99,105,143$ & 8 & 4 \\
\hline Total & 6 & 26 & 32 & 17 \\
\hline
\end{tabular}

The types from outside of the RHA-panel are in bold 


\section{Results of nPCR amplicons sequencing}

In total, 48 samples positive with the universal probe in RHA were subjected to sequencing. Only for 19 of those $(39.6 \%)$ it was possible to determine the Beta-HPV genotype. According to Berkhout and others (Berkhout et al., 1995) and Surentheran and others (Surentheran et al., 1998), not only nucleotide but also amino acid sequences were used for the genotype assessment. If the sequence homology was above 90\% compared to any known type, it was considered as an established type, while 75-90\% homology meant the so-called "related type". When comparing the obtained sequences to those available in the NCBI database, the presence of 25 types from the panel detected by RHA as well as the presence of types from outside the RHA panel but belonging to the genus Betapapillomavirus was uncovered. The results of direct sequencing of nPCR amplicons were presented in Table 2. In these 19 biopsies analysed, 25 established Beta-HPV genotypes were revealed. Among these, fifteen types represented the RHA panel (HPV5, $8,9,12,15,20,25,36,80)$. In 14 samples with genotypes confirmed by RHA, the direct sequencing allowed detection of extra 13 genotypes, including 8 genotypes from outside of the RHA panel. Among the 161 related types, 55\% represented types from the RHA panel. The most common ones were: HPV99, 110, 113, 122. Only in three out of 19 samples analysed, a single genotype was present (HPV25, HPV105 or HPV151).

In one case, $100 \%$ homology was observed between the analysed sequence and the corresponding reference HPV12 type.

Among the five samples positive only with the universal probe in RHA, 32 different Beta-HPV sequences were identified, including six established types (HPV5, $15,20,36,124,152)$. In four of these samples, BetaHPVs from outside the panel were detected (Table 3).

Sequencing of eight samples positive for HPV5 by RHA confirmed the presence of this oncogenic type only in two cases (together with HPV36 and HPV143 types). The presence of HPV8 was not confirmed in the amplicons sequenced.

\section{DISCUSSION}

Studies of the incidence of skin Beta-HPVs conducted in samples from patients with NMSC from different countries depend on the sensitivity and specificity of the methods used.

In the present study, we evaluated the molecular methods of determining the presence of Beta-HPVs in the skin from cancerous or precancerous lesions and from neighbouring clinically healthy skin. Based on the previous studies (Berkhout et al., 1995; Wieland et al., 2000), freshly frozen biopsies were selected for the analysis. Our research began in 2006 and lasted for ten years, which allowed us to collect enough material to evaluate the methods used. The choice of the two-step nPCR method according to Berkhout et al. (1995) was dictated by a rather low amount of the virus in the changed tissues, as described by other authors (Stockfleth et al., 2004; Wieland et al., 2000). Next methods used were the specific one-step PCRs for HPV5 and HPV8 and a novel commercial test for genotyping. In the selected pool of specimens, the study was extended by direct sequencing of nPCR amplicons.

The detection threshold of the nPCR method used by us turned out to be similar to that given by Berkhout and others (Berkhout et al., 1995) and Meyer and others
(Meyer et al., 2000). First, nPCR and hybridization with universal probes were compared. The nPCR proved to be significantly less sensitive than RHA at its relatively high specificity. This fact and only $80 \%$ agreement of these two tests possibly result from the amplification of a longer fragment of the DNA strand by nPCR than in RHA (374-389 bp and 117 bp, respectively) as well as the application of degenerate CP-primers for nPCR in contrast to RHA with nine nondegenerate primers. Other authors also pointed to such differences, e.g. in their study, Meyer and others (Meyer et al., 2000) underlined the fact that the application of nondegenerate primers increases the sensitivity of the PCR. Similar results were reported by de Koning and others (de Koning et al., 2006), who emphasized statistically lower sensitivity of nPCR with slightly modified CP primers compared to RHA. Furthermore, nPCR detects 19 types of Beta-HPVs, while RHA can also identify additional types, such as HPV47, 75, 76, 80, 92, 93, 96. This fact also contributes to the underestimation of the results obtained with this nPCR method. To increase the possibility of HPV detection using PCR, other research groups employed several additional primer pairs (Meyer et al., 2000; Stockfleth et al., 2004), slight modifications in primer sequences or PCR conditions (Harwood et al., 1999; Berkhout et al., 2000) as well as the application of primers derived from the viral E region (de Koning et al., 2006; Gheit et al., 2007).

In contrast to the previous reports (de Koning et al., 2006; Bouwes Bavinck et al., 2010), by using the commercial RHA test, we revealed the presence of BetaHPVs less often, because only in about $40 \%$ of the tested biopsies. These discrepancies may result from different clinical diagnosis and material used in the study (not only skin biopsies but also eyebrow hair), as well as different epidemiology of Beta-HPV infections in different geographic regions of the world. In our studies, in contrast to the ones mentioned above, in the group of patients diagnosed with NMSC, BCC dominated, not SCC.

Moreover, in $5 \%$ of the samples tested by the RHA, we obtained positive results only with universal probes without the identification of specific types. Mackintosh and others (Mackintosh et al., 2009) showed similar results in $13 \%$ of their examined samples. Such results indicated the presence of unspecified genotypes (from outside of the tested panel) from the Beta-HPV species or, otherwise, too low viral load for genotyping. Obtaining HPV confirmations for types 38, 92 and 96 without a positive reaction with universal probes can be explained by the inactivity of these probes with the aforementioned types of virus, which was confirmed by de Koning and others (de Koning et al., 2006).

In the reports by Zakrzewska and others (Zakrzewska et al., 2012) and Mackintosh and others (Mackintosh et al., 2009), similarly to the current study, both beta-1 and beta-2 species predominated among the studied types. This fact is understandable due to the highest number of HPV types in these species. The profile of genotypes found in our studies is very similar to many earlier publications. The most common types, 23, 93 and 24, also occurred as dominant in the previous research (de Koning et al., 2009; Bouwes Bavinck et al., 2010).

In the majority of tests carried out with the RHA, the presence of HPV47 was not confirmed and similar negative results were also obtained in this study. Only Bouwes Bavinck and others (Bouwes Bavinck et al., 2010) and Zakrzewska and others (Zakrzewska et al., 2012) reported the presence of HPV47 in the tested material 
from Southern Europe in a small $(1-4 \%)$ share of all genotypes. Considering the high sensitivity of the RHA for HPV47 (10 copies per assay), according to de Koning and others (de Koning et al., 2006), the above fact can only be explained by the absence or very low load of HPV47 in the diagnostic samples.

The results obtained using reverse hybridization, reported in all above-discussed studies, as well as our data, confirmed the frequent presence of mixed types of BetaHPVs in various clinical material tested.

Like other authors, we focused on two oncogenic Beta-HPV types. However, in contrast to most of the previous studies, there were few HPV5 or HPV8 identified in our study using not only specific PCR but also the RHA method. The most similar results using the RHA were obtained by Mackintosh and others (Mackintosh et al., 2009) who showed the presence of HPV5 and HPV8 DNA in $6.8 \%$ and $2.5 \%$ of the biopsies analysed, respectively. In material collected mainly from BCC, Zakrzewska and others (Zakrzewska et al., 2012) confirmed the presence of both high-risk HPV types in $18 \%$ and $10 \%$ of samples, respectively. Using nPCR with specific primers for HPV8, Pfister and others (Pfister et al., 2003) detected infections with this type in up to $60 \%$ of biopsies from the skin with pre-cancerous lesions in Polish patients. Comparing our results with the abovequoted data, a rather low load of these oncogenic BetaHPVs in materials collected from our patients cannot be excluded. Therefore, the nPCR with specific primers, more sensitive than single-step PCR, seems to be more useful. Moreover, considering the large number of multiple infections, underestimation of single types associated with a low load of their DNA may not be ruled out (de Koning et al., 2007).

The analysis of the results of direct sequencing was possible only in about $40 \%$ of the samples tested due to overlapping peaks in the obtained fluorogram, possibly indicating the presence of multiple HPV types in the samples assayed ( $\mathrm{Li}$ et al., 2013). By the sequencing, additional types were shown, other than the ones detected only by the hybridization method as in the report by Gheit and others (Gheit et al., 2007). Most of the sequences analysed yielded results indicating the presence of several types of Beta-HPV species, both from the RHA panel as well as from outside of this panel. In agreement with previously presented studies (de Koning et al., 2006; Gheit et al., 2007), the majority of analysed sequences indicated the presence of HPVs belonging to the beta- 1 and beta- 2 species. Sequencing of samples that showed a positive reaction only with the universal probes in RHA revealed the presence of not only types from outside of the RHA panel but also the presence of other types that should be detected by hybridization. The reason for this, which other authors also pointed out (Antonsson et al., 2013), might be too low load of individual types of viruses and the presence of viral coinfections. In contrast to the RHA, among the analysed sequences, the presence of HPV type 47 was demonstrated in two samples tested as the related type (75$90 \%$ homology). The detection failures for RHA and sequencing of HPV5 and 8 might have been due to the missing certain types in direct sequencing of amplicons derived from broad-spectrum PCR (Forslund et al., 1999; Meyer et al., 2000). Given the low viral load in tissues with NMSC (one copy per 100-400 cells) (Weissenborn et al., 2009), only dominant types could be amplified and sequenced in samples with multiple Beta-HPV infections.

A significant spread of Beta-HPVs in the environment and in the skin, low viral load in the tissues, a large va- riety of viral genotypes and high frequency of multiple infections justify the need to use not only sensitive but also specific methods for the detection of viral infections. On the other hand, the lack of adopted standardization rules, the use of methods of different sensitivity and primers of different specificity make it difficult and sometimes impossible to compare the obtained results. In the present work, the results of genotyping obtained by RHA were confirmed and extended to identify additional types by the nPCR method with subsequent sequencing as well as the PCR with primers specific for potentially oncogenic Beta-HPV types. In our opinion, the statistically-supported compliance of all methods used for the detection and genotyping of Beta-HPVs turned out to be only moderate. In view of the above, as well as taking into account the results obtained, we are advocates of assessing the presence and dissemination of a viral infection by not only one detection method but by extending the diagnostic possibilities to use other methods.

Perhaps in the future, sensitive, established and standardized diagnostic methods will allow assessing the risk of developing cancer and contribute to the inclusion of prevention as well as targeted therapy to prevent BetaHPV-related cancers.

\section{Conflict of interest}

The authors declare that they have no conflict of interest.

\section{REFERENCES}

Antonsson A, Michael KM, Pawlita M, Lehmann MD, Nindl I (2013) Detection and typing of cutaneous human papillomavirus types - a comparison of three different methods. J. Virol. Methods 189: 305310. https://doi.org/10.1016/j.jviromet.2012.10.010

Berkhout RJ, Bouwes Bavinck JN, ter Schegget J (2000) Persistence of human papillomavirus DNA in benign and (pre)malignant skin lesions from renal transplant recipients. J. Clin. Microbiol. 38: 20872096. PMCID: PMC86734

Berkhout RJ, Tieben LM, Smits HL, Bavinck JN, Vermeer BJ, ter Schegget J (1995) Nested PCR approach for detection and typing of epidermodysplasia verruciformis-associated human papillomavirus types in cutaneous cancers from renal transplant recipients. J. Clin. Microbiol. 33: 690-695. PMCID: PMC228015

Bouvard V, Baan R, Straif K, Grosse Y, Secretan B, El Ghissassi F, Benbrahim-Tallaa L, Guha N, Freeman C, Galichet L, Cogliano V (2009) WHO International Agency for Research on Cancer Monograph Working Group. A review of human carcinogens - Part B: biological agents. Lancet Oncol. 10: 321-322. https://doi. org/10.1016/s1470-2045(09)70096-8

Bouwes Bavinck JN, Neale RE, Abeni D, Euvrard S, Green AC, Harwood CA, de Koning MN, Naldi L, Nindl I, Pawlita M, Pfister H, Proby CM, Quint WG, ter Schegget J, Waterboer T, Weissenborn S, Feltkamp MC; EPI-HPV-UV-CA group (2010) Multicenter study of the association between betapapillomavirus infection and cutaneous squamous cell carcinoma. Cancer Res. 70: 9777-9786. https:// doi.org/10.1158/0008-5472.CAN-10-0352

Brink AA, Lloveras B, Nindl I, Heideman DA, Kramer D, Pol R, Fuente MJ, Meijer CJ, Snijders PJ (2005) Development of a generalprimer-PCR-reverse-line-blotting system for detection of beta and gamma cutaneous human papillomaviruses. J. Clin. Microbiol. 43: 5581-5587. https://doi.org/10.1128/JCM.43.11.5581-5587.2005

de Jong SJ, Imahorn E, Itin P, Uitto J, Orth G, Jouanguy E, Casanova JL, Burger B (2018) Epidermodysplasia verruciformis: inborn errors of immunity to human beta-papillomaviruses. Front. Microbiol. 9: 1222. https://doi.org/10.3389/fmicb.2018.01222

de Koning MN, Quint W, Struijk L, Kleter B, Wanningen P, van Doorn LJ, Weissenborn SJ, Feltkamp M, terSchegget J (2006) Evaluation of a novel highly sensitive, broad-spectrum PCR-reverse hybridization assay for detection and identification of betapapillomavirus DNA. J. Clin. Microbiol. 44: 1792-1800. https://doi. org/10.1128/JCM.44.5.1792-1800.2006

de Koning MN, Struijk L, Bavinck JN, Kleter B, terSchegget J, Quint WG, Feltkamp MC (2007) Betapapillomaviruses frequently persist in the skin of healthy individuals. J. Gen. Virol. 88: 1489-1495. https://doi.org/10.1099/vir.0.82732-0 
de Koning MN, Weissenborn SJ, Abeni D, Bouwes Bavinck JN, Euvrard S, Green AC, Harwood CA, Naldi L, Neale R, Nindl I, Proby CM, Quint WG, Sampogna F, ter Schegget J, Struijk L, Wieland U, Pfister HJ, Feltkamp MC; EPI-HPV-UV-CA group (2009) Prevalence and associated factors of betapapillomavirus infections in individuals without cutaneous squamous cell carcinoma. J. Gen. Virol. 90: 1611-1621. https://doi.org/10.1099/vir.0.010017-0

Forslund O (2007) Genetic diversity of cutaneous human papillomaviruses. J. Gen. Virol. 88: 2662-2669. https://doi.org/10.1099/ vir. $0.82911-0$

Forslund O, Antonsson A, Nordin P, Stenquist B, Hansson BG (1999) A broad range of human papillomavirus types detected with a general PCR method suitable for analysis of cutaneous tumours and normal skin. J. Gen. Virol. 80: 2437-2443. https://doi. org/10.1099/0022-1317-80-9-2437

Forslund O, Ly H, Higgins G (2003) Improved detection of cutaneous human papillomavirus DNA by single tube nested 'hanging droplet' PCR. J. Virol. Methods 110: 129-136. https://doi.org/ 10.1016/ s0166-0934(03)00109-5

Fuchs PG, Iftner T, Weninger J, Pfister H (1986) Epidermodysplasia verruciformis-associated human papillomavirus 8: genomic sequence and comparative analysis. J. Virol. 58: 626-634. PMCID: PMC252953

Gheit T (2019) Mucosal and cutaneous human papillomavirus infections and cancer biology. Front. Oncol. 9: 355. https://doi, org/10.3389/fonc. 2019.00355

Gheit T, Billoud G, de Koning MN, Gemignani F, Forslund O, Sylla BS, Vaccarella S, Franceschi S, Landi S, Quint WG, Canzian F, Tommasino M (2007) Development of a sensitive and specific multiplex PCR method combined with DNA microarray primer extension to detect Betapapillomavirus types. J. Clin. Microbiol. 45: 25372544. https://doi.org/10.1128/JCM.00747-07

Harwood CA, Spink PJ, Surentheran T, Leigh IM, de Villiers EM, McGregor JM, Proby CM, Breuer J (1999) Degenerate and nested PCR: a highly sensitive and specific method for detection of human papillomavirus infection in cutaneous warts. J. Clin. Microbiol. 37: 3545-3555. PMCID: PMC85688

Jablonska S, Dabrowski J, Jakubowicz K (1972) Epidermodysplasia verruciformis as a model in studies on the role of papovaviruses in oncogenesis. Cancer Res. 32: 583-589. PMID: 5061309

Kawase M, Orth G, Jablonska S, Blanchet-Bardon C, Rueda LA, Favre M (1996) Variability and phylogeny of the L1 capsid protein gene of human papillomavirus type 5: contribution of clusters of nonsynonymous mutations and of a 30-nucleotide duplication. Virology 221: 189-198. https://doi.org/10.1006/viro.1996.0365

Li J, Pan Y, Xu Z, Wang Q, Hang D, Shen N, Liu M, Zhang C, Abliz A, Deng Q, Cai H, Ke Y (2013) Improved detection of human papillomavirus harbored in healthy skin with FAP6085/64 primers. J. Virol. Methods 193: 633-638. https://doi.org/10.1016/j.jviromet.2013.06.026

Mackintosh LJ, de Koning MN, Quint WG, ter Schegget J, Morgan IM, Herd RM, Campo MS (2009) Presence of beta human papillomaviruses in nonmelanoma skin cancer fromorgan transplant recipients and immunocompetent patients in the West of Scotland. Br. J. Dermatol. 161: 56-62. https://doi.org/10.1111/j.13652133.2009.09146.x

Meyer T, Arndt R, Christophers E, Stockfleth E (2000) Frequency and spectrum of HPV types detected in cutaneous squamous- cell carcinomas depend on the HPV detection system: a comparison of four PCR assays. Dermatology 201: 204-211. https://doi. org/10.1159/000018489

Pfister H, Fuchs PG, Majewski S, Jablonska S, Pniewska I, Malejczyk M (2003) High prevalence of epidermodysplasia verruciformisassociated human papillomavirus DNA in actinic keratoses of the immunocompetent population. Arch. Dermatol. Res. 295: 273-279. https://doi.org/10.1007/s00403-003-0435-2

Rollison DE, Viarisio D, Amorrortu RP, Gheit T, Tommasino M (2019) An emerging issue in oncogenic virology: the role of beta human papillomavirus types in the development of cutaneous squamous cell carcinoma. J. Virol. 93: 1003-1018. https://doi. org/10.1128/JVI.01003-18

Stockfleth E, Nindl I, Sterry W, Ulrich C, Schmook T, Meyer T (2004) Human papillomaviruses in transplant-associated skin cancers. Dermatol. Surg. 30: 604-609. https://doi.org/10.1016/j.ygyno.2019.03.101

Surentheran T, Harwood CA, Spink PJ, Sinclair AL, Leigh IM, Proby CM, McGregor JM, Breuer J (1998) Detection and typing of human papillomaviruses in mucosal and cutaneous biopsies from immunosuppressed and immunocompetent patients and patients with epidermodysplasia verruciformis: a unified diagnostic approach. J. Clin. Pathol. 51: 606-610. https://doi.org/10.1136/jcp.51.8.606

Szostek S, Klimek M, Zawilinska B, Kosz-Vnenchak M (2008) Genotype-specific human papillomavirus detection in cervical smears. Acta Biochim. Pol. 55: 687-692. PMID: 19015776

Szostek S, Zawilinska B, Kopec J, Kosz-Vnenchak M (2009) Herpesviruses as possible cofactors in HPV-16-related oncogenesis. Acta Biochim. Pol. 56: 337-342. PMID: 19499088

Tommasino M (2017) The biology of beta human papillomaviruses. $\mathrm{Vi}$ rus Res. 231: 128-138. https://doi.org/10.1016/j.virusres.2016.11.013

Tommasino M (2019) HPV and skin carcinogenesis. Papillomavirus Res. 7: 129-131. https://doi.org/10.1016/j.pvr.2019.04.003

Van Doorslaer K, Chen Z, Bernard HU, Chan PKS, DeSalle R, Dillner J, Forslund O, Haga T, McBride AA, Villa LL, Burk RD, Ictv Report Consortium (2018) ICTV virus taxonomy profile: papillomaviridae. J. Gen. Virol. 99: 989-990. https://doi.org/10.1099/ jgv.0.001105

Viarisio D, Gissmann L, Tommasino M (2017) Human papillomaviruses and carcinogenesis: well-established and novel models. Curr. Opin. Virol. 26: 56-62. https://doi.org/10.1016/j.coviro.2017.07.014

Weissenborn SJ, Neale R, de Koning MN, Waterboer T, Abeni D, Bouwes Bavinck JN, Wieland U, Pfister HJ; EPI-HPV-UV-CA Group (2009) Prevalence and multiplicity of cutaneous beta papilloma viruses in plucked hairs depend on cellular DNA input. $J$. Virol. Methods 161: 280-283. https://doi.org/10.1016/j.jviromet.2009.06.024

Wieland U, Ritzkowsky A, Stoltidis M, Weissenborn S, Stark S, Ploner M, Majewski S, Jablonska S, Pfister HJ, Fuchs PG (2000) Communication: papillomavirus DNA in basal cell carcinomas of immunocompetent patients: an accidental association? J. Invest. Dermatol. 115: 124-128. https://doi.org/10.1046/j.1523-1747.2000.00015.x

Zakrzewska K, Regalbuto E, Pierucci F, Arvia R, Mazzoli S, Gori A, de Giorgi V (2012) Pattern of HPV infection in basal cell carcinoma and in perilesional skin biopsies from immunocompetent patients. Virol. J. 9: 309. https://doi.org/10.1186/1743-422X-9-309

Zur Hausen H (2000) Papillomaviruses causing cancer: evasion from host-cell control in early events in carcinogenesis. J. Natl. Cancer Inst. 92: 690-698. https://doi.org/10.1093/jnci/92.9.690 